\title{
The immediate psychological effects of Coronavirus Disease 2019 on medical and non-medical students in China
}

\author{
Lin $\mathrm{Xie}^{1} \cdot \mathrm{Hong} \mathrm{Luo}^{2} \cdot \mathrm{Mei} \mathrm{Li}^{3} \cdot$ Wenjie Ge ${ }^{1} \cdot$ Bingyu Xing ${ }^{1} \cdot$ Qunfang Miao $^{1}$
}

Received: 13 March 2020 / Revised: 30 July 2020 / Accepted: 31 August 2020/Published online: 10 September 2020

(C) Swiss School of Public Health (SSPH+) 2020

\begin{abstract}
Objectives To investigate the immediate psychological effects of Coronavirus Disease 2019 (COVID-19) on medical and non-medical students.

Methods An online survey of 805 medical students and 1900 non-medical students was conducted from Feb 4, 2020 to Feb 7, 2020, in China. The questionnaire measured the subjective estimated severity of COVID-19, the impact of the outbreak, and the levels of anxiety and depression of both medical and non-medical students.

Results Medical students estimated COVID-19 to be more serious and disastrous than non-medical students, while they scored lower than non-medical students on the Impact of Event Scale-Revised (IES-R), and less severe anxiety and depression than non-medical students. The students experienced greater impact from the outbreak and a higher rate of anxiety and depression with increased time focusing on the outbreak. The difference in psychological effects between medical and non-medical students was further enlarged when focusing time was prolonged.

Conclusions The immediate psychological effects of COVID-19 on medical and non-medical students exhibit different characteristics. The outcome of this study provides implication that providing accurate and transparent information about the epidemic and appropriate COVID-19-based knowledge in accessible ways will contribute to the public's mental health during the outbreak.
\end{abstract}

Keywords COVID-19 · Psychological effect · Impact of event · Anxiety · Depression · Medical student

Qunfang Miao

m18668071616@163.com

Lin Xie

zjuxl@126.com

Hong Luo

luohong@hznu.edu.cn

Mei Li

83181721@qq.com

Wenjie Ge

1377021730@qq.com

Bingyu Xing

993097193@qq.com

1 Medical School, Hangzhou Normal University, Room 508, Building No.8 in Shenyuan, No.2318 of Yuhangtang Rd, Yuhang District, Hangzhou 311121, China

2 Department of Clinical Psychology, The Affiliated Hospital of Hangzhou Normal University, Hangzhou, China

3 Psychological Counseling Center, Hangzhou Normal University, Hangzhou, China

\section{Introduction}

Coronavirus Disease 2019 (COVID-19) has spread rapidly and widely to all the 31 provinces in the mainland of China since January 28, 2020 (NHC of the People's Republic of China 2020a). More than 30 thousand people had been confirmed to be infected with COVID-19 by February 7, 2020, including about seven hundreds deaths resulting from the disease and six thousands severe cases, according to the official website of the National Health Commission (NHC of the People's Republic of China, 2020b). Humanto-human transmissions were identified, and the number of confirmed cases and suspected cases increased rapidly. People experienced stress in response to information on COVID-19, changes in daily routines and the uncertainty regarding the future of the epidemic. The stress would lead to a psychological impact and might result in hazards that exceed the consequences of the epidemic itself (Bao et al. 2020). Investigating the psychological effects of COVID- 
19 would help establish a scientific and effective mental health support system.

In the past twenty years, several emergent public health events, such as SARS, MERS, Ebola and H1N1, have occurred around the world. Many studies have been conducted to explore the psychological effects of these epidemic outbreaks on various groups of people, demonstrating that the stress people experienced results in immediate mental health problems, such as anxiety and depression, as well as post-traumatic stress disorder and vicarious traumatization with long-term effects (Van Bortel et al. 2016). Infected patients and their contacts have been closely studied. They suffered from life-threatening infections and witnessed other patients die, resulting in stress during and after the outbreak (Lee et al. 2007; Rabelo et al. 2016), and some felt shame and guilt by being stigmatized by their communities (Cheung 2015; Person et al. 2004). Medical staffs have also been closely studied. Witnessing the traumatic consequences of an epidemic, medical staff experience substantial distress, mainly due to the fear of infection, loss of control and spread of the virus (Tambo et al. 2014; Wong et al. 2005). Among medical staff, those who performed MERS-related tasks in higher-risk environments experienced greater psychological impacts than those working in other environments (Lee et al. 2018; Matsuishi et al. 2012). Furthermore, they felt helpless and guilt for their inability to save patients and felt frustrated by long working hours, work overload and the lack of protective equipment (van Bortel et al. 2016).

Compared with patients, their contacts and the medical staff, the general population experienced a relatively lowrisk environment. However, they also experienced fear and worried about being infected (Blakey et al. 2015; Cheung 2015; Person et al., 2004; Taylor and Asmundson 2004). They obtained information through official and unofficial channels and lacked the ability to distinguish accurate information from misinformation. They had not been trained on efficient methods of prevention. It was thought that they felt fear of the unknown (Ki 2014). The medical students have been professional trained, and they have more medical knowledge than others. Several studies have demonstrated that knowledge of the causes, prevention and treatment of a disease helps decrease the worry about an outbreak (Patel et al. 2018; Siddle et al. 2016). In addition, the medical staff improved their knowledge after educational intervention, simultaneously increasing the confidence to face the epidemic outbreak (Patel et al. 2018; Siddle et al. 2016). However, to date, there is still much unknown about COVID-19. It is still unclear whether historical medical science knowledge and skills will help them manage their fear of subsequent epidemics. On the other hand, a study conducted after a SARS outbreak showed that medical students felt more anxious and depressed than other students due to the large number of medical staff and medical students being infected (Wong et al. 2007). It is also unclear whether empathy toward the medical staff working in a high-risk environment increases stress of medical students. Thus, it is important to investigate the psychological effects of the epidemic outbreak on medical students to determine the effects of knowledge on the emotional reactions to the outbreak, which would help to increase understanding of the psychological mechanisms of stress during epidemics, and to construct an effective psychological support system for different population groups.

As summarized above, the present study attempted to measure the subjective impact of the outbreak, and the levels of anxiety and depression of both medical and nonmedical students by an online survey, as well as the university students' assessment of how risky the COVID-19 outbreak was. Based on previous studies, we hypothesized that medical students would experience a greater psychological impact than non-medical students.

\section{Methods}

\section{Participants}

The study was conducted from Feb 4, 2020, to Feb 7, 2020, and surveyed 2705 undergraduate students (Table 1). All the students were living outside Hubei, and none of them was infected by COVID-19. They voluntarily participated in this study, which was approved by the university's Institutional Review Board. They received the linkage of the online survey by instant messengers and were instructed to complete it online. After a participant completed the survey, he or she received their score, an estimation of their mental health status and a suggestion about what they should do if the results of the questionnaire revealed that they were anxious or depressed.

\section{Questionnaire}

The survey included four parts. The first part collected the basic information of the students, with major, sex, place of residence and time spent focusing on relevant information each day. The second part collected the participants' views on COVID-19, with four descriptions of the epidemic: (1) COVID-19 is serious. It is difficult to prevent and lacks effective treatments. (2) COVID-19 will become widespread for a long time because it is highly infectious. (3) The outbreak will have catastrophic consequences. (4) The outbreak can be managed in society as a whole. These four items were developed based on risk perception theory, attempting to investigate the university students' 
Table 1 The basic information of the online-survey participants (China, Feb 4 to Feb 7, 2020)

\begin{tabular}{lcc}
\hline & Number of people & Ratio (\%) \\
\hline Major & & \\
Medical & 805 & 29.75 \\
Non-medical & 1900 & 70.24 \\
Sex & & \\
Male & 608 & 22.48 \\
Female & 2097 & 77.52 \\
Region & & \\
Provincial capital city & 494 & 18.26 \\
Prefecture-level city & 580 & 21.44 \\
County-level city & 674 & 24.92 \\
Town & 383 & 14.16 \\
Country & 574 & 21.22 \\
Time focusing on COVID-19-related information & & 42.51 \\
$<1$ h & 1150 & 47.17 \\
$1-3$ h & 1276 & 10.32 \\
$>3$ h & 279 & \\
\hline
\end{tabular}

assessment of how risky the COVID-19 outbreak was, in terms of probabilistic estimates of the degree of situational uncertainty and how controllable that uncertainty was (Liu et al. 2006). The participants were instructed to choose the option they agreed with.

The third part used the Chinese version of the Impact of Event Scale-Revised (IES-R) to measure subjective responses to a specific traumatic event (Guo et al. 2007; Weiss 2007). It was recognized as one of the earliest selfreport tools to evaluate post-traumatic stress. The 22-item scale includes three subscales: the avoidance subscale, the intrusion subscale and the hyperarousal subscale. Higher scores indicate more serious states. The Chinese version of the IES-R has been shown to be reliable and valid, with Cronbach's $\alpha$ of 0.89 and a split-half reliability of 0.93 .

The fourth part used the Chinese version of the 7-item Generalized Anxiety Disorder Scale (GAD-7), which is a valid and efficient tool to identify self-reported anxiety (He et al. 2010; Spitzer et al. 2006). Cutoff points of 5, 10 and 15 on the GAD-7 were interpreted as representing mild, moderate and severe levels of anxiety, respectively. The Cronbach's $\alpha$ of the Chinese version of the GAD-7 was 0.90 , and the test-retest reliability was 0.86 . This part also used the Chinese version of the depression module of the Patient Health Questionnaire (PHQ-9), a frequently used and well-standardized measure of self-reported depression (BIAN et al. 2009; Kroenke and Spitzer 2002). Cutoff points of 5, 10 and 15 on the PHQ-9 were interpreted as representing mild, moderate and severe levels of depression, respectively. Cronbach's $\alpha$ of the Chinese version of the PHQ-9 was 0.86, and the test-retest reliability was 0.95 .

\section{Statistical analysis}

The data collected through the survey were analyzed by SPSS 18.0 (SPSS Inc., USA). The Chi-square test was used to compare the time spent focusing on COVID-19-related information, and the views on COVID-19 between medical and non-medical students. For the IES-R, a two-way ANOVA with two between-subjects factors (major: medical/non-medical, time spent focusing on information about COVID-19: $<1 \mathrm{~h} / 1-3 \mathrm{~h} />3 \mathrm{~h}$ ) was used to compare the average scores of the avoidance subscale, the intrusion subscale and the hyperarousal subscale. For the GAD-7 and PHQ-9, the Mann-Whitney $U$ test was used to compare severe levels of anxiety and depression. Two-way ANOVA with two between-subjects factors (major: medical/nonmedical, focusing time on information about COVID19: $<1 \mathrm{~h} / 1-3 \mathrm{~h} />3 \mathrm{~h}$ ) was also used to compare the total score of GAD-7 and PHQ-9. A simple effect analysis was performed for further comparisons between the major groups and the focusing time groups when the interaction of these two factors was significant. Statistical significance was set at $p<0.05$.

\section{Results}

\section{Views on COVID-19}

There were significant effects of medical/non-medical major on the description "COVID-19 is serious. It is difficult to prevent and lacks effective treatments" (ratio of agree: medical $47.33 \%$, non-medical $52.68 \%, p=0.012$ ) and "The outbreak will have catastrophic consequences" 
Fig. 1 The avoidance subscale scores of IES-R (the Chinese version of the Impact of Event Scale-Revised) of medical and non-medical students $(* * * p<0.001, * * p<0.01$, $\left.{ }^{*} p<0.05\right)$. Medical students showed lower avoidance than non-medical students, with a significant difference in the groups whose focusing time was longer than one hour (China, Feb 4 to Feb 7, 2020)

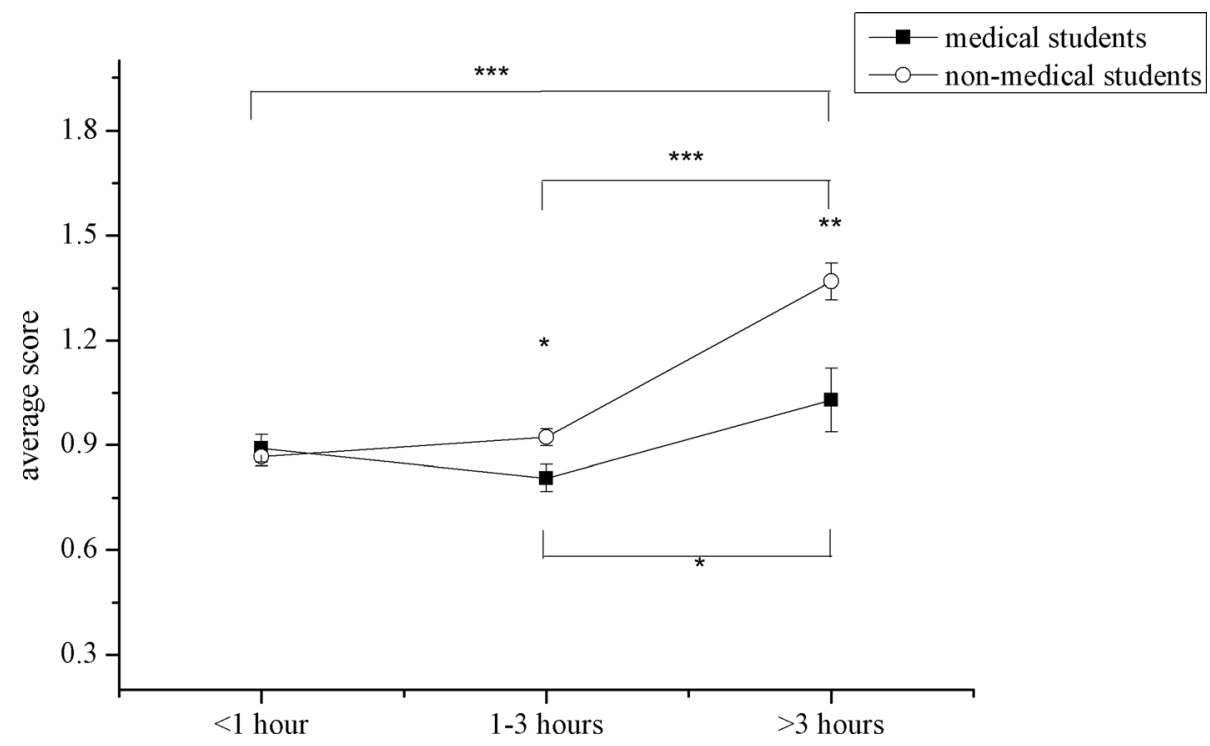

(ratio of agree: medical $81.24 \%$, non-medical $85.42 \%$, $p=0.008$ ) but no significant effects on "COVID-19 will become widespread for a long time because it is highly infectiousness" (ratio of agree: medical 21.49\%, nonmedical $19.95 \%, p=0.375$ ) and "The outbreak can be managed by society as a whole" (ratio of agree: medical $62.61 \%$, non-medical $63.58 \%, p=0.632$ ).

\section{The impact of event}

The medial and non-medical students showed no significant difference in the time spent focusing on COVID-19related information $\left(\chi^{2}=4.63, p=0.201\right)$. The means and SEs of the IES-R scores are presented in Figs. 1, 2, 3. There were significant effects of major on the subjective evaluation of avoidance $(F=12.12, p=0.001)$, intrusion $(F=7.98, \quad p=0.005)$ and hyperarousal $(F=6.14$, $p=0.013$ ), as well as significant effects of focusing time on the subjective evaluation of avoidance $(F=17.89$, $p<0.001)$, intrusion $(F=45.40, p<0.001)$ and hyperarousal $(\mathrm{F}=29.32, \mathrm{p}<0.001)$. There was also an interaction between major and focusing time on avoidance $(\mathrm{F}=5.67, \mathrm{p}=0.003)$, intrusion $(\mathrm{F}=5.98, \mathrm{p}=0.003)$ and hyperarousal $(F=3.38, p=0.034)$. Simple effect analysis revealed that medical students who spent more than three hours focusing on COVID-19 had higher scores on all three subscales than those who focused on COVID-19 for less than three hours (avoidance subscale: $1-3 \mathrm{~h}$ vs. $>3 \mathrm{~h}$ : $p=0.024$, intrusion subscale: $<1 \mathrm{~h}$ vs. $>3 \mathrm{~h}: p<0.001$, $1-3 \mathrm{~h}$ vs. $>3 \mathrm{~h}: p<0.001$, hyperarousal subscale: $<1 \mathrm{~h}$ vs. $>3 \mathrm{~h}: p=0.007,1-3 \mathrm{~h}$ vs. $>3 \mathrm{~h}: p=0.002$ ). For non-medical students, the group who spent more than three hours focusing on COVID-19 had higher scores on the avoidance subscale $(<1 \mathrm{~h}$ vs. $>3 \mathrm{~h}: p<0.001,1-3 \mathrm{~h}$ vs. $>3 \mathrm{~h}: p<0.001)$ and hyperarousal subscale $(<1 \mathrm{~h}$ vs. $>3$ h: $p<0.001,1-3$ h vs. $>3$ h: $p<0.001$ ) than the other groups. In the intrusion subscale, the longer the focusing time was, the higher non-medical students scored ( $<1$ h vs. $1-3$ h: $p=0.002,<1$ h vs. $>3$ h: $p<0.001$, $1-3 \mathrm{~h}$ vs. $>3 \mathrm{~h}: p<0.001)$. Simple effect analysis of major also revealed that medical students showed lower avoidance than non-medical students when focusing time was longer than one hour $(1-3 \mathrm{~h}: p=0.012,>3 \mathrm{~h}$ : $p=0.001)$, and they reported lower intrusion $(p=0.002)$ and hyperarousal $(p=0.008)$ than non-medical students when focusing time was longer than three hours.

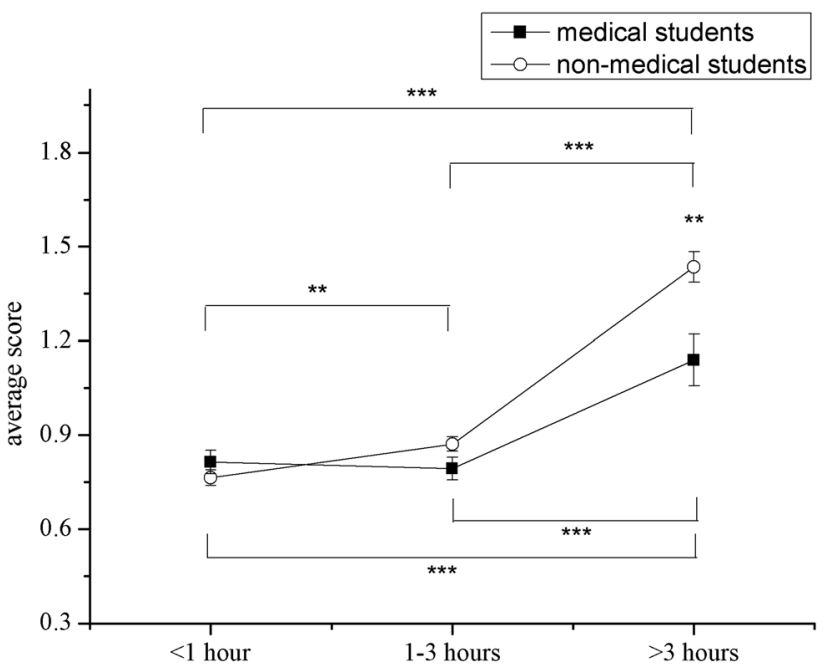

Fig. 2 The intrusion subscale scores of IES-R (the Chinese version of the Impact of Event Scale-Revised) of medical and non-medical students $\left(* * * p<0.001,{ }^{* *} p<0.01, * p<0.05\right)$. The score increased with focusing time in non-medical students. Medical students showed lower intrusion than non-medical students, with a significant difference in the groups whose focusing time was longer than three hours (China, Feb 4 to Feb 7, 2020) 

subscale scores of IES-R (the Chinese version of the Impact of Event Scale-Revised) of medical and non-medical students $(* * * p<0.001$, $* * p<0.01, * p<0.05)$. Medical students showed lower hyperarousal than non-medical students, with a significant difference in the groups whose focusing time was longer than three hours (China, Feb 4 to Feb 7, 2020)
Fig. 3 The hyperarousal
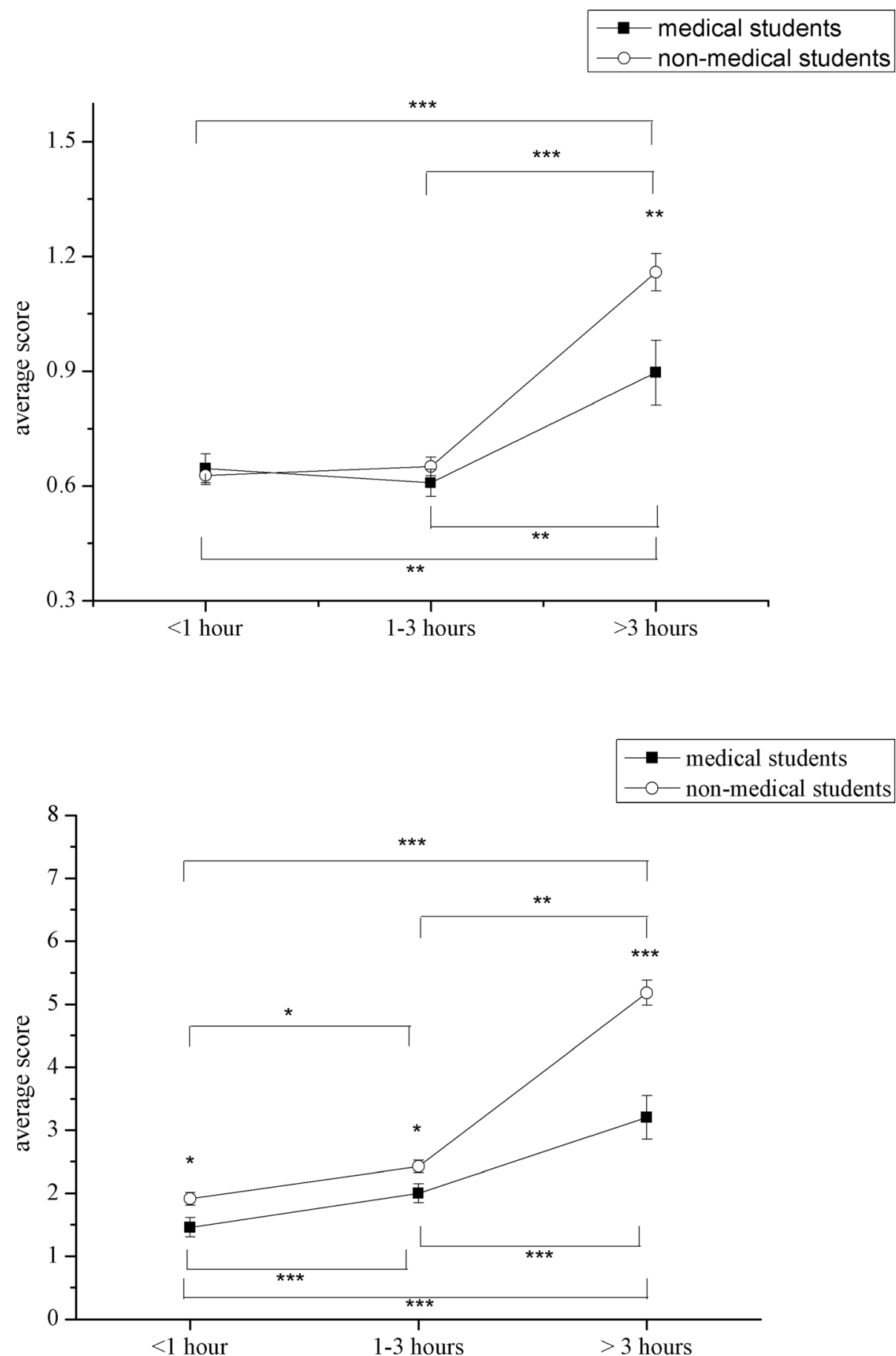

Fig. 4 The GAD-7 (the Chinese version of the 7-item Generalized Anxiety Disorder Scale) scores for medical and non-medical students $(* * * p<0.001, * * p<0.01$, $* p<0.05)$. The self-reported score of both medical and nonmedical students increased with times spent focusing on information about COVID-19 (Coronavirus Disease 2019). Medical students scored lower than non-medical students (China, Feb 4 to Feb 7, 2020)

\section{Anxiety and depression}

The ratios of each severe levels of anxiety and depression were calculated (GAD-7: none $85.21 \%$, minimal $13.29 \%$, moderate $1.24 \%$, severe $0.25 \%$; PQH-9: none $81.79 \%$, minimal $13.74 \%$, moderate $3.58 \%$, severe $0.90 \%$ ). The Mann-Whitney $U$ test revealed that there were significant differences in severe levels reported on the GAD-7
$(\mathrm{Z}=-2.40, \quad p=0.016)$ and PHQ-9 $\quad(Z=-3.54$, $p<0.001)$ between medical and non-medical students.

The means and SEs of the scores on the GAD-7 and PHQ-9 are presented in Figs. 4 and 5. There was a significant effect of major on the GAD-7 scores $(F=35.84$, $p<0.001$ ), as well as a significant effect of focusing time on the GAD-7 scores $(F=64.44, p<0.001)$. An interaction of major and focusing time was found in the GAD-7 
Fig. 5 The PHQ-9 (the Chinese version of the depression module of the Patient Health Questionnaire) scores for medical and non-medical students $(* * * p<0.001$, $* * p<0.01, * p<0.05)$. The focusing time on COVID-19 (Coronavirus Disease 2019) information significantly affected the self-reported score. The scores of medical students were lower than those of nonmedical students in the group who spent more than three hours focusing on COVID-19 (China, Feb 4 to Feb 7, 2020)

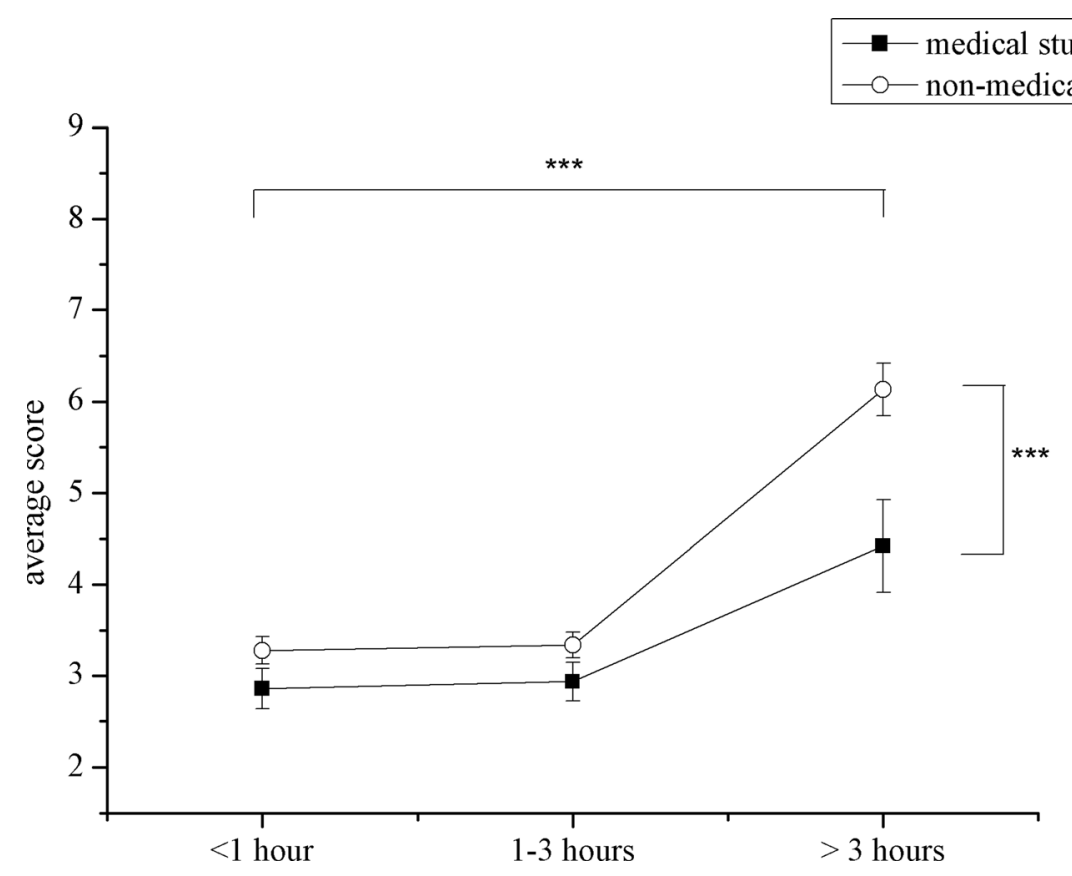

scores $(F=6.67, p=0.001)$. Simple effect analysis revealed that the GAD-7 scores increased with focusing time in both medical students ( $<1 \mathrm{~h}$ vs. $1-3 \mathrm{~h}: p=0.011$, $<1 \mathrm{~h}$ vs. $>3 \mathrm{~h}: p<0.001,1-3 \mathrm{~h}$ vs. $>3 \mathrm{~h}: p=0.002$ ) and non-medical students $(<1 \mathrm{~h}$ vs. $1-3 \mathrm{~h}: p<0.001$, $<1$ h vs. $>3$ h: $p<0.001,1-3$ h vs. $>3$ h: $p<0.001$ ). Medical students scored lower than non-medical students in all three focus time groups $(<1 \mathrm{~h}: p=0.014,1-3 \mathrm{~h}$ : $p=0.018,>3 \mathrm{~h}: p<0.001)$. For the PHQ-9 scores, there was a significant effect of major $(F=13.54, p<0.001)$, as well as a significant effect of focusing time $(F=25.51$, $p<0.001)$. However, there was no interaction of major and focusing time for PHQ-9 scores $(F=2.29, p=0.102)$. The post hoc analysis revealed that the students whose spent more than three hours focusing on information about COVID-19 scored higher than the other two groups $(<1 \mathrm{~h}$ vs. $>3$ h: $p<0.001,1-3$ h vs. $>3$ h: $p<0.001$ ).

\section{Discussion}

The present study was focused on examining the immediate psychological effects of COVID-19 on medical and non-medical students. The findings of this study demonstrated that medical students judge the epidemic outbreak to be more serious than non-medical students and predicted more disastrous consequences. However, medical students experienced a lower subjective impact of the epidemic and fewer mental health problems than non-medical students. Considering the variable time spent focusing on information about COVID-19, the results show that students had a greater impact from the outbreak and experience higher rates of anxiety and depression with increased focusing time. It should be noted that the difference in the psychological effects experienced by medical and non-medical students was further enlarged when focusing time was prolonged.

A series of studies investigating the psychological impacts on medical staff working in higher-risk environments and lower-risk environments revealed that distress about epidemics is related with the risk of infection (Lee et al. 2018; Matsuishi et al. 2012). However, the general population of medically healthy people who experience only lower-risk environments can also experience psychological effects that result in distress and hysteria during an epidemic outbreak (Taylor and Asmundson 2004). During the outbreak of Ebola, a term called "Fearbola" was created to refer to negative experiences such as fear and anxiety among the general population (James 2014). The results of the present study likewise show that a considerable number of participants experienced various degrees of anxiety and depression. Approximately $17 \%$ of all the participants felt anxious, and 29\% felt depressed. These psychological impacts on the general population were thought to be due to fear of the unknown (Ki 2014). Thus, knowledge of the disease responsible for an outbreak might be an important factor to moderate the psychological impact. Medical students are generally thought to have more knowledge of epidemics than other college students. Comparing medical and non-medical students, the results of the present study show that medical students experience a lower impact of events and less severe anxiety and 
depression than non-medical students. This was consistent with the findings of previous studies on the psychological effects of SARS on college students in Hong Kong (Chua et al. 2004; Wong et al. 2004a). In cognitive models of pathological anxiety, corrective information plays an important role in psychological therapy (Abramowitz et al. 2019), which might further support the role of knowledge in alleviating psychological distress in response to epidemics. Knowledge would help individuals accurately estimate the severity and risk of contamination from an outbreak, which would lead to appropriate emotions and behaviors. Therefore, in response to the panic over Ebola, the American Psychological Association suggested that people "get the facts" from their physician, a local or state public health agency or national and international resources (APA 2014).

It should be noted that the medical students in the present study judged the COVID-19 to be more serious than non-medical students. This result suggests that knowledge of the severity of an epidemic does not affect mental health, but professional knowledge of the causes, transmission routes, prevention and treatment of infectious diseases may be what matters. A previous study on the psychological impact of SARS demonstrated that vulnerability/loss of control was an important variable in explaining the level of distress of healthcare workers (Wong et al. 2005). During the outbreak of Ebola in Africa, some medical staff showed negative attitudes and perceptions toward the patients because of their lack of training and preparation (Otu et al. 2016). Several studies demonstrated that after educational intervention, the medical staff increased their knowledge and felt they were better prepared to face the epidemic outbreak (Patel et al. 2018; Siddle et al. 2016). Thus, it can be seen that providing upto-date and adequate information about an epidemic and self-protection methods is important to counteract feelings of anxiety and depression.

That is not to say, however, gathering more information was better for mental health. The information available during an epidemic outbreak is always excessive and is sometimes inadequate and sensationalist. Several studies on media coverage during the outbreak of SARS confirmed this (Dixon 2003; Rezza et al. 2004). A previous study of university students showed that the overdone of media using did not affect their anxiety or their knowledge of SARS. While in the present study, both medical and nonmedical students experienced more negative feelings with prolonged time focusing on COVID-19-related information. This inconsistency may come from the information dissemination approaches in these two periods. Currently, in an era of information explosion, people can obtain much more information in more ways, with increasing accessibility to informal information. Trust of informal information was thought to be a factor in epidemic-related anxiety (Liao et al. 2011; Ro et al. 2017). The results of the present study also show a growing difference between medical and non-medical students with increased time spent focusing on COVID-19-related information. It seems possible that because medical students have more basic knowledge of medical science, they are more able to determine the reliability of information. Thus, with more received information, more differences were observed between the two groups. It should also be noted that excessive information may lead to anxiety, and anxiety may also lead to an intensive search for information (Baumgartner and Hartmann 2011). Therefore, the increased anxiety and depression associated with prolonged focusing time may be explained by the fact that the students who experienced more anxiety and depression due to the epidemic tended to spend more time focusing on related information.

There is abundant room for further progress in investigating the current topic. First, the participants in this study were all in good health and from regions outside Hubei, the most intense areas of COVID-19 infection. Therefore, it is no surprise that the results of the present study are contrary to those of the study conducted in 2003 during the SARS epidemic in Hong Kong (Wong et al. 2007). Since several medical students were infected during that outbreak, it was claimed that the medical students in Hong Kong perceived a higher risk of contracting SARS during the outbreak (Wong et al. 2004b, 2007). Future studies on the psychological effects of COVID-19 on medical and non-medical students from a higher-risk environment are therefore recommended. Second, further studies that account for the effects of knowledge on feelings of anxiety and depression will need to be undertaken. It is important to investigate the effects of various degrees and categories of information and exploring mediators between knowledge and emotional experience, such as coping style. It would improve understanding of the psychological mechanism of epidemic effects and be instructive for guiding educational interventions. In addition, a further study with more focus on the sustainability of these psychological effects is suggested. Many studies have shown that survivors, their contacts and healthcare workers suffer psychiatric disorders such as post-trauma stress disorder and depression after outbreaks. However, the long-term psychological effects on the health concept and health behavior of the general population are still lacking. Moreover, since most medical students have clear career objectives and most of them will work in medical-related positions after graduation, it is meaningful to investigate the effects of what they saw and heard about the experience of medical staff in engaging in saving lives from COVID-19. It would also 
make sense to track the career path of these medical students after graduation.

\section{Conclusion}

Medical students experienced lower psychological impact of COVID-19 than non-medical students, although they thought the epidemic more serious. Time spent focusing on epidemic-related information affected the psychological effects of COVID-19, and the difference in the psychological effects experienced by medical and non-medical students increased with time prolonged. The outcome of this study suggests that providing accurate and transparent information about the epidemic and providing appropriate COVID-19-based knowledge training in accessible ways will contribute to the public's mental health during the outbreak.

Funding No funding was received for this study.

\section{Compliance with ethical standards}

Conflict of interest The authors declare that there is no conflict of interest with any individual/organization for the present work.

Ethical standards The present study was approved by the Institutional Review Board of Hangzhou Normal University. The principal Investigator was Qunfang Miao, associate professor of Hangzhou Normal University. All the subjects voluntarily participated in the online survey, which clearly state the aim of the survey and the survey was anonymous. Before the questionnaire, it was clearly stated that they did not need to complete it if they did not want to, and they can quit anytime during their filling in.

\section{References}

Abramowitz JS, Deacon BJ, Whiteside SP (2019) Exposure therapy for anxiety: principles and practice. Guilford Publications, New York

American Psychological Association (2014) Managing your fear about Ebola. https://www.apa.org/helpcenter/ebola-fear. Accessed 15 February 2015

Bao Y, Sun Y, Meng S et al (2020) 2019-nCoV epidemic: address mental health care to empower society. The Lancet 395:e37-e38

Baumgartner SE, Hartmann T (2011) The role of health anxiety in online health information search. Cyberpsychol Behav Soc Netw 14:613-618

Bian C, He X, QIAN J, et al (2009) The reliability and validity of a modified patient health questionnaire for screening depressive syndrome in general hospital outpatients. J Tongji Univ (Med Sci) 30(5):136-140

Blakey SM, Reuman L, Jacoby RJ et al (2015) Tracing "Fearbola": psychological predictors of anxious responding to the threat of Ebola. Cognit Therapy Res 39:816-825

Cheung EY (2015) An outbreak of fear, rumours and stigma: psychosocial support for the Ebola Virus Disease outbreak in West Africa. Intervention 13:70-76
Chua SE, Cheung V, Cheung C et al (2004) Psychological effects of the SARS outbreak in Hong Kong on high-risk health care workers. Can J Psychiat 49:391-393

Dixon B (2003) Editors sound the SARS alarm bells. Curr Biol 13:R339-R340

Guo S, Xin Z, Geng L (2007) Reliability and validity of Chinese version of the impact of event scale-revised. Chin J Clin Psychol 15:15

He X, Li C, Qian J et al (2010) Reliability and validity of a generalized anxiety disorder scale in general hospital outpatients. Shanghai Arch Psychiat 22:200-203

James JJ (2014) Fearbola. Disaster Med Public Health Preparedness $8: 465-466$

Ki M (2014) What do we really fear? The epidemiological characteristics of Ebola and our preparedness. Epidemiology and health 36:e2014014

Kroenke K, Spitzer RL (2002) The PHQ-9: a new depression diagnostic and severity measure. Psychiatric annals 32:509-515

Lee AM, Wong JG, McAlonan GM et al (2007) Stress and psychological distress among SARS survivors 1 year after the outbreak. Can J Psychiat 52:233-240

Lee SM, Kang WS, Cho A-R et al (2018) Psychological impact of the 2015 MERS outbreak on hospital workers and quarantined hemodialysis patients. Compr Psychiat 87:123-127

Liao Q, Cowling BJ, Lam WWT et al (2011) The influence of socialcognitive factors on personal hygiene practices to protect against influenzas: using modelling to compare avian A/H5N1 and 2009 pandemic A/H1N1 influenzas in Hong Kong. Int J Behav Med 18:93-104

Liu J, Zhou G, Huang H (2006) A review of research on risk perception. Psychol Sci 29(2):370-372

Matsuishi K, Kawazoe A, Imai H et al (2012) Psychological impact of the pandemic (H1N1) 2009 on general hospital workers in Kobe. Psychiat Clin Neurosci 66:353-360

National Health Commission of the People's Republic of China (2020a) Update on epidemic situation of novel coronavirusinfected pneumonia by 24:00 on Jan. 28, 2020.https://www.nhc. gov.cn/xcs/yqtb/202001/1c259a68d81d40abb939a0781c1fe237. shtml. Accessed on July 30, 2020

National Health Commission of the People's Republic of China (2020b) Update on epidemic situation of novel coronavirusinfected pneumonia by $24: 00$ on Feb. 7, 2020.https://www.nhc. gov.cn/xcs/yqtb/202002/6c305f6d70f545d59548ba17d79b8229. shtml. Accessed on July 30, 2020

Otu A, Ebenso B, Okuzu O, Osifo-Dawodu E (2016) Using a mHealth tutorial application to change knowledge and attitude of frontline health workers to Ebola virus disease in Nigeria: a before-andafter study. Hum Resour Health 14(5):5

Patel I, Guy J, Han Y et al (2018) Effects of Ebola Virus Disease education on student health professionals. Curr Pharm Teach Learn 10:651-656

Person B, Sy F, Holton K et al (2004) Fear and stigma: the epidemic within the SARS outbreak. Emerg Infect Dis 10:358

Rabelo I, Lee V, Fallah MP et al (2016) Psychological distress among ebola survivors discharged from an ebola treatment unit in monrovia, liberia - a qualitative study. Front Public Health. https://doi.org/10.3389/fpubh.2016.00142

Rezza G, Marino R, Farchi F et al (2004) SARS epidemic in the press. Emerg Infect Dis 10(2):381-382

Ro J-S, Lee J-S, Kang S-C et al (2017) Worry experienced during the 2015 Middle East respiratory syndrome (MERS) pandemic in Korea. PLoS ONE 12(3):e0173234

Siddle J, Tolleson-Rinehart S, Brice J (2016) Survey of emergency department staff on disaster preparedness and training for Ebola virus disease. Am J Disaster Med 11:5-18 
Spitzer RL, Kroenke K, Williams JB et al (2006) A brief measure for assessing generalized anxiety disorder: the GAD-7. Arch Intern Med 166:1092-1097

Tambo E, Ugwu EC, Ngogang JY (2014) Need of surveillance response systems to combat Ebola outbreaks and other emerging infectious diseases in African countries. Infect Dis Poverty 3:29

Taylor S, Asmundson GJ (2004) Treating health anxiety: a cognitivebehavioral approach. Guilford Press, New York

Van Bortel T, Basnayake A, Wurie F et al (2016) Psychosocial effects of an Ebola outbreak at individual, community and international levels. Bull World Health Organ 94:210

Weiss DS (2007) The impact of event scale: revised. In: Crosscultural assessment of psychological trauma and PTSD. Springer, Boston

Wong JG, Cheung EP, Cheung V et al (2004) Psychological responses to the SARS outbreak in healthcare students in Hong Kong. Med Teach 26:657-659
Wong T-w, Lee C-k, Tam W et al (2004) Cluster of SARS among medical students exposed to single patient. Hong Kong Emerg Infect Dis 10:269

Wong TW, Yau JK, Chan CL et al (2005) The psychological impact of severe acute respiratory syndrome outbreak on healthcare workers in emergency departments and how they cope. Eur J of Emerg Med 12:13-18

Wong TW, Gao Y, Tam WWS (2007) Anxiety among university students during the SARS epidemic in Hong Kong. Stress Health J Int Soc Investig Stress 23:31-35

Publisher's Note Springer Nature remains neutral with regard to jurisdictional claims in published maps and institutional affiliations. 